\title{
Myotonia dystrophica with heart involvement: an electron microscopic study of skeletal, cardiac, and smooth muscle
}

\author{
R. M. LUdATSCHER, H. KERNER, S. AMIKAM, AND B. GELlEI \\ From the Departments of Pathology and Cardiology, The Technion School of Medicine \\ and Rambam Medical Center, Haifa, Israel
}

SUMMARY The electron microscopic features of the striated skeletal muscle, the striated cardiac muscle, and the smooth muscle from a woman who had been suffering for many years from myotonia dystrophica with cardiac involvement are described. The skeletal muscle was studied at two different stages of the disease. In the first material the main changes consisted of centrally situated nuclei, disorganisation of the sarcomeres, and focal disruption of the Z-line. The satellite cells were well represented. Three years later atrophy and degenerative, necrotic changes of the skeletal muscle were evident. The satellite cells were absent. Few changes were seen in the striated cardiac muscle. These consisted of slight interstitial fibrosis and large accumulations of mitochondria with intramitochondrial dense granules. The smooth muscle cells of the oesophagus showed disorientated filaments and mild degenerative changes. It is concluded that the skeletal muscle was more severely affected than the other types of muscle.

Myotonia dystrophica is an hereditary autosomal disease, chiefly characterised by progressive weakness and atrophy of the skeletal muscles.

Heart involvement in patients with myotonia dystrophica has been known since 1920 (Maas and Zondek, 1920). The morphological changes in the myocardium and, to a lesser extent, the heart conduction system have been reported previously (Cannon, 1962; Church, 1967; Bulloch et al., 1967; Rausing, 1972; Uemura et al., 1973).

The fine structural alterations in the striated muscle and in the muscle spindles in myotonia dystrophica are now well documented (Aleu and Afifi, 1964; Daniel and Strich, 1964; Klinkerfuss, 1967; Mussini et al., 1970; Schotland, 1970; Swash and Fox, 1975; Ludatscher et al., 1976; Maynard et al., 1977).

It appears that an ultrastructural description of the smooth muscle in myotonia dystrophica is lacking, although smooth muscle dysfunction is clinically well known, and the changes have been reported based on manometric, radiological (Harvey et al., 1965), and histological (Pruzanski and Huvos, 1967) studies.

In the present study the ultrastructural changes of

Received for publication 29 March 1978 the skeletal, cardiac, and smooth muscles of a woman who had been suffering for many years from myotonia dystrophica are reported.

\section{Case report}

A 50-year-old woman, married and the mother of a 21-year-old son, was admitted to hospital for recurrent attacks of loss of consciousness. She had suffered from these attacks for the last four years, having been diagnosed an epileptic. She received regular treatment with barbiturates and phenytoin sodium with no improvement. Her past history revealed that at the age of 30 she had undergone bilateral extraction of the eye lenses because of cataracts. During the last 15 years she had had progressive difficulty in walking. Physical examination revealed a pale, fat woman with the face and gait typical of myotonia. Ptosis of both upper eyelids and nasal speech were evident. There was wasting of the distal muscles of the extremities. The tendon reflexes were absent. Laboratory examination revealed transient hypocalcaemia (calcium $6 \cdot 5-7.5 \mathrm{mg} / 100 \mathrm{ml}$; $1.6-1.9 \mathrm{mmol} / \mathrm{l}$ ) with normal serum phosphate values. Electromyography showed myotonic discharges. A chest $x$-ray film suggested moderate cardiomegaly. 
The electrocardiogram showed second-degree atrioventricular block with a ventricular rate of 40 per minute. The patient was diagnosed as myotonia dystrophica with a high degree of atrioventricular block. Her late father, brother, and sister had all suffered from the same disease. A permanent transvenous endocardial pacemaker was implanted and therapy with procainamide and hydantoin was introduced. Follow-up examination revealed that the patient had suffered no more Adams-Stokes attacks, but skeletal muscle weakness was present. During her last admission, which occurred four months before death, the patient complained of severe pain in the left foot related to gangrene of the fingers. Above-knee amputation of the left leg was performed. Two days later she died from pulmonary embolism. Necropsy revealed marked, generalised atherosclerosis in addition to right pulmonary embolism and thrombosis of the right auricle.

\section{Material and methods}

Striated muscle was obtained on two occasions. The first specimen was removed from the pectoralis major under local anaesthesia with $1 \%$ procaine solution. Two years and nine months later necropsy material was taken three hours after death. Skeletal muscle samples were taken from the following muscles: left biceps, right extensor digitorum, right gastrocnemius, trapezius, intercostal, and diaphragmatic. Cardiac muscle was removed from both ventricles and atria. For the study of smooth muscle, fragments from the aorta, the lower third of the oesophagus, and the small intestine were excised. All the material was processed in the same manner. A large part of the tissue was fixed in $10 \%$ formalin for light microscopy. A smaller part was immediately fixed in cold $3 \%$ glutaraldehyde, post-fixed with $2 \%$ osmium tetroxide, dehydrated with alcohol, and embedded in Epon. Sections $1 \mu \mathrm{m}$ thick were stained with toluidine blue and examined with the light microscope. The smooth muscle layers of the aorta, oesophagus, and intestine were selected for thin sectioning. All thin sections were stained with lead citrate and uranyl acetate and examined with a Zeiss $9 \mathrm{~S}$ electron microscope.

\section{Results}

STRIATED SKELETAL MUSCLE

The biopsy material from the pectoralis major, which had been examined three years before death, showed variations in the size of the myofibres, but no marked atrophy of the muscle cells was noticed. The main ultrastructural changes consisted of an increased number of sarcolemmal nuclei with centrally located nuclei; changes in the orientation of the myofilaments; and focal disruption of the Z-line. Occasionally clumps of degenerated myofilaments were noted. Satellite cells were present in the extrafusal muscle fibres (Fig. 1) and in the intrafusal muscle cells of the muscle spindle as well. The alterations of the muscle spindle consisted of degenerative changes of the intrafusal muscle cells with deep-cell invaginations and consecutive formation of intranuclear pockets. Three years later the ab-

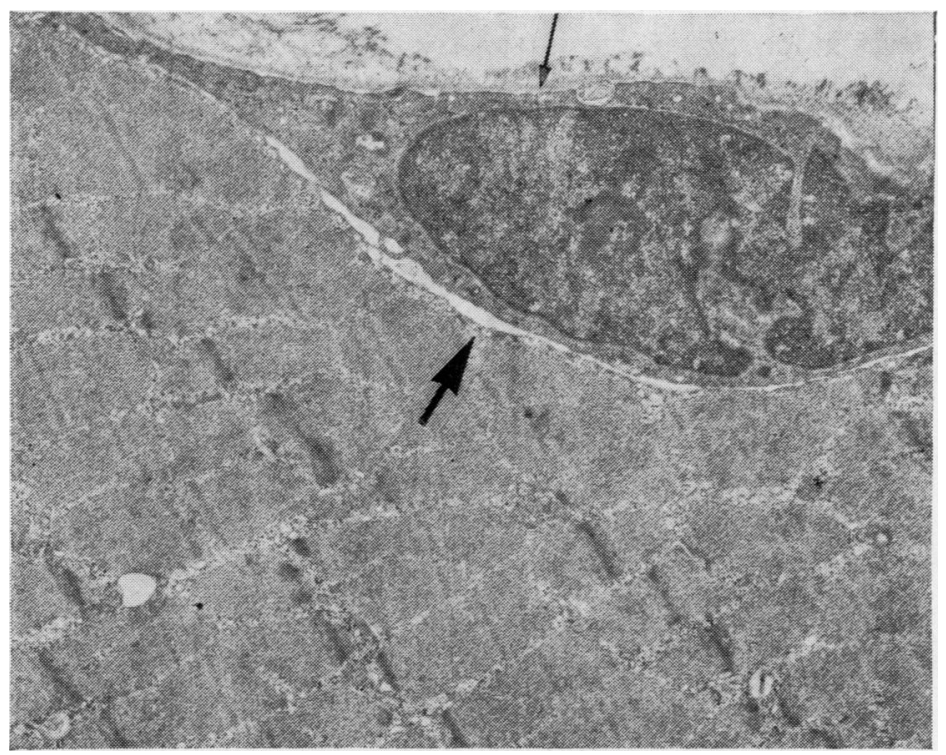

Fig. 1 Muscle cell from the pectoralis major shows a regular sarcomere pattern. $A$ satellite cell with prominent nucleus is enclosed by the sarcolemma (arrow). Note the narrow space between the striated muscle cell and the satellite cell (thick arrow). $\times 9750$ 


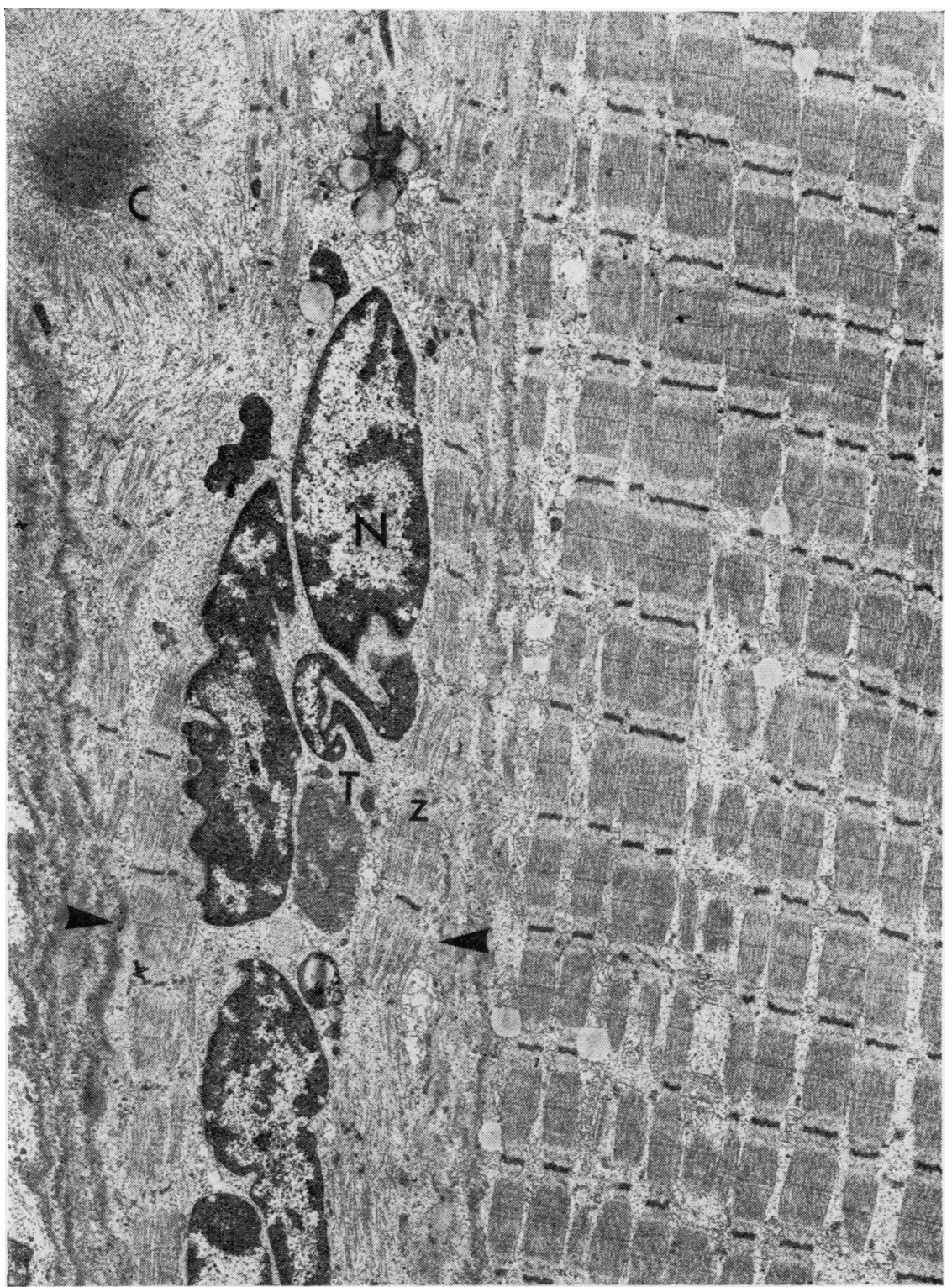

Fig. 2 Gastrocnemius. Three longitudinally sectioned cells are seen. The muscle cell in the centre (between arrows) is atrophic and largely occupied by a chain of nuclei $(N)$. Loss of Z-line at Z. Cytoplasmic body at C. Lipofuscin body at L.T $=$ compact narrow tubules. $\times 5700$

normalities of the striated muscle had progressed in a striking manner. Severe changes were seen in the gastrocnemius, extensor digitorum, and biceps muscles. Atrophic muscle cells averaged $8-10 \mu \mathrm{m}$ in diameter and were seen in the vicinity of regular sized muscle fibres (Fig. 2). A large part of the muscle was composed of adipose tissue with a few atrophic muscle cells dispersed here and there. In such areas collagen fibrils, small blood vessels, and nerve structures outnumbered the myofibres. There were different grades of fibre degeneration in a given section. In the less affected muscle cells the Z-line was interrupted for short segments, and focal lack of myofilaments occurred in these places. Glycogen 
granules, lipid vacuoles, or lipofuscin bodies occupied the degenerated regions (Fig. 2). In other atrophic cells there was massive loss of myofilaments with total disintegration of the Z-line. Chains of marginal nuclei and centrally located nuclei were frequent (Fig. 2). Cytoplasmic bodies were noted. These consisted of a dense, central core surrounded by a lighter area composed of radiating myofilaments (Fig. 2). An unusual formation of compact, narrow tubules appeared very occasionally in the atrophic degenerated cell (Fig. 2). Collagen fibrils adhered to the sarcolemma and usually filled the narrow space between adjacent muscle cells (Fig. 2). Ringen fibres were common in all muscles. Folding and reduplication of the sarcolemmal membrane was often seen. Empty vacuoles or lipid-containing vacuoles were usually associated with an excess of glycogen granules. No satellite cells were seen in the muscles described above. The intercostal and diaphragmatic muscles showed centrally located nuclei, ringen fibres, and mild degenerative changes. However, the changes in the intercostal muscles were less marked than in the muscles of the extremities.

\section{STRIATED CARDIAC MUSCLE}

The heart weighed $350 \mathrm{~g}$ and the left ventricle was slightly hypertrophic. The light microscopic sections showed irregular sized muscle cells with moderately enlarged cells in the left ventricle. Areas of adipose tissue and areas of fibrosis were dispersed among the myocardial cells of both sides of the heart. The amount of adipose tissue was more marked in the right ventricle.

Ultrastructurally, there were no differences in the morphology of the myocardial cells of the right and left ventricles. Variation in the size of the muscle cells was a constant finding. The sarcomere pattern was disorganised owing to rupture and distortion of the Z-line (Fig. 3). In longitudinally orientated sections, the disrupted myofibrils were fusiformly

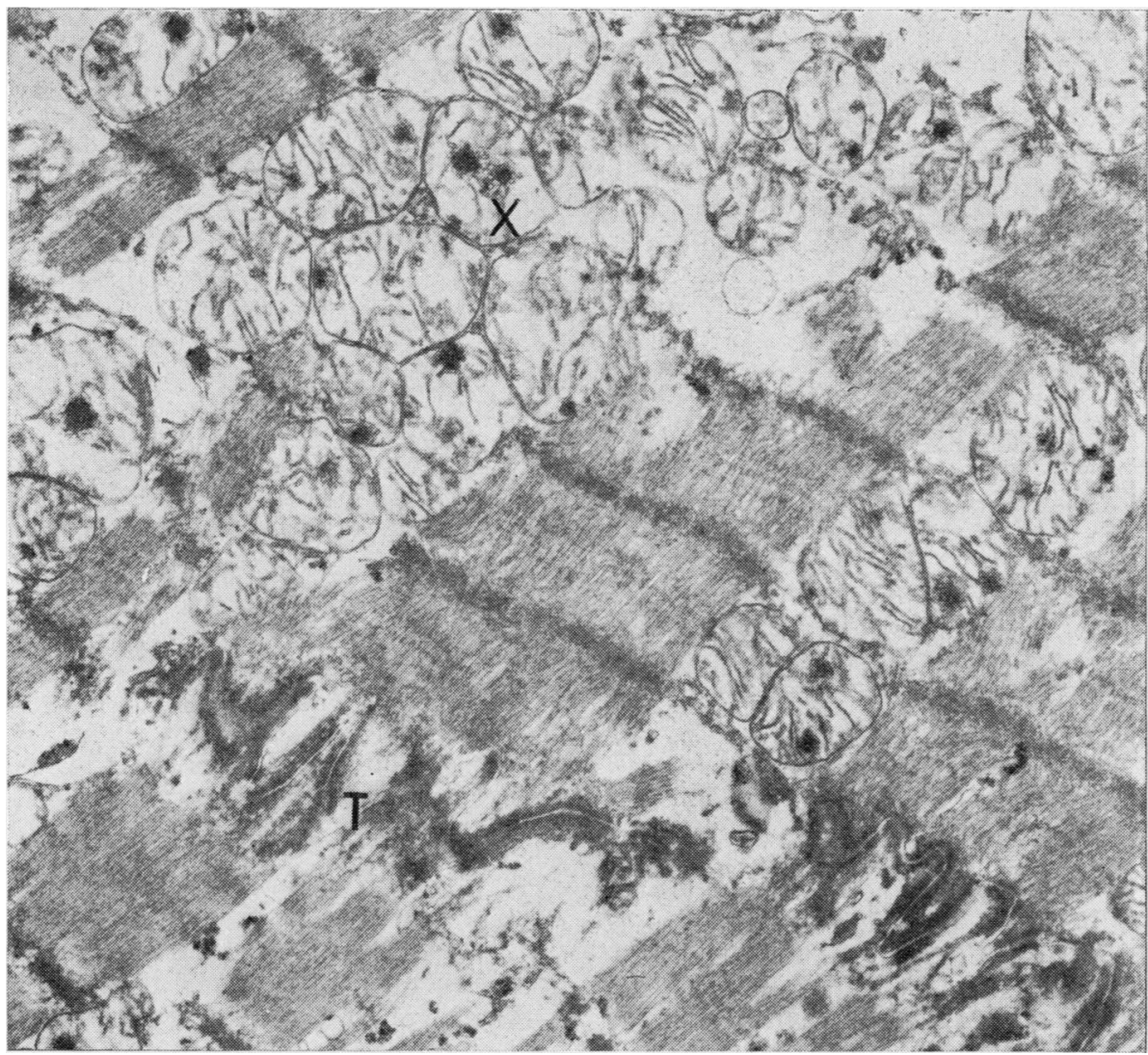

Fig. 3 Myocardium. Area with disrupted sarcomeres is occupied by crowded mitochondria showing electron-dense granules $(X) . T=$ intercalated disc. $\times 14700$ 
shaped and had tapered ends. There was focal loss of myofilaments, and large masses of mitochondria occupied the areas where myofilaments were absent (Fig. 3). The mitochondria appeared either in small crowded groups or were randomly dispersed (Fig. 3). Disrupted mitochondrial cristae and one or two dense granules were seen in many mitochondria. The mitochondrial granules were $300-400 \AA$ in diameter and occupied no favourite place inside the mitochondrion (Fig. 3). No distinct membrane was binding the mitochondrial granules, which sometimes were associated with remnants of cristae. In many muscle cells the area occupied by mitochondria was impressive. Empty, dilated vacuoles and dense bodies were dispersed at random. There was no evidence of alteration of the intercalated disc. An increase in the amount of collagen fibrils in the interstitial space between adjacent myocardial cells was obvious.

\section{SMOOTH MUSCLE}

The ultrastructural aspect of the smooth muscle cells was different in the tunica media of the aorta from that in the wall of the digestive tract.

\section{Aorta}

The smooth muscle cells of the aortic media were irregularly shaped (Fig. 4). The plasma membrane was undulated and featured cytoplasmic extensions.

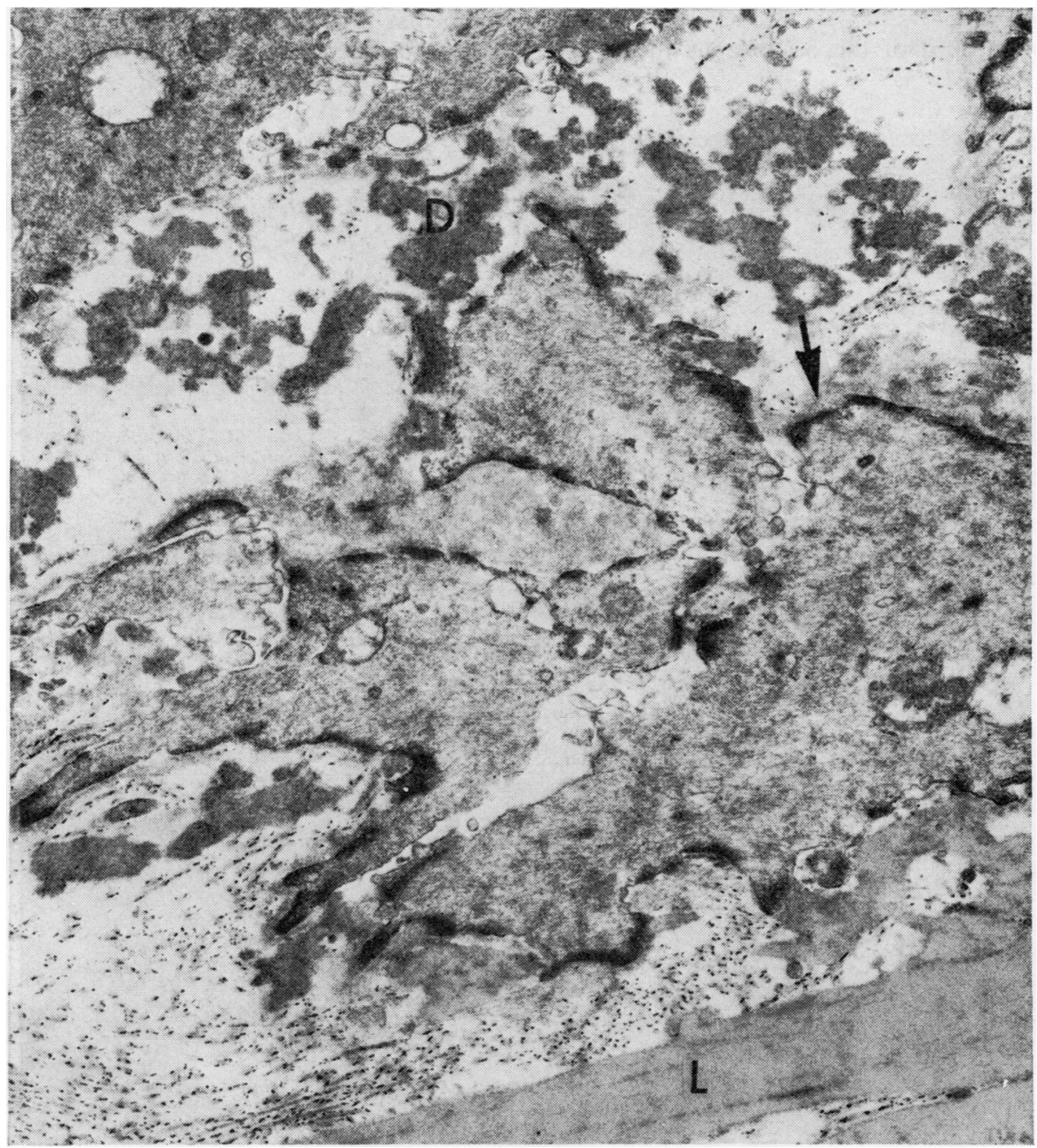

Fig. 4 Aorta. Cytoplasmic densities are seen along the plasma membrane of the smooth muscle cells (thick arrow). $L=$ elastic lamella. Irregularly shaped dense material is seen between muscle cells $(D) . \times 14700$ 
The cytoplasmic filaments were arranged in a disorderly way. Cytoplasmic densities were obviously scattered along the inner aspects of the plasma membrane (Fig. 4). A small number of pinocytotic vesicles was noticed. Lipid inclusions occurred in the cytoplasm of some cells. In many areas the elastic lamellae of the aorta appeared as organised straight bands, $2 \mu \mathrm{m}$ thick, and sometimes the elastic tissue was contiguous with the muscle cell membrane (Fig. 4). In some areas a relatively large amount of irregular sheets of dense amorphous material was dispersed in the interstitial space between the muscle cells (Fig. 4). Collagen fibrils were scattered among the smooth muscle cells.

\section{Oesophagus}

In the lower third of the oesophagus the smooth muscle cells were of an elongated shape and were regularly arranged parallel to each other (Fig. 5). Their diameter ranged from 2 to $4 \mu \mathrm{m}$. The cells were enclosed almost completely by basement membrane (Fig. 5). Along the cell surface there were small areas devoid of both cell membrane and basal lamina. The cytoplasm was filled with longitudinally orientated filaments. However, in many cells disorganisation in the arrangement of the filaments occurred (Fig. 5). Most myofilaments measured $50 \AA$ in diameter. Thicker myofilaments ( $90 \AA$ in diameter) were clearly seen (Fig. 5). Cytoplasmic densities were prominent along the inner sites of the cell membrane and along the myofilaments as well. In some cells glycogen granules accumulated adjacent to the myofilaments, thus creating glycogen bodies (Fig. 5). Pinocytotic vesicles were numerous. Collagen fibrils

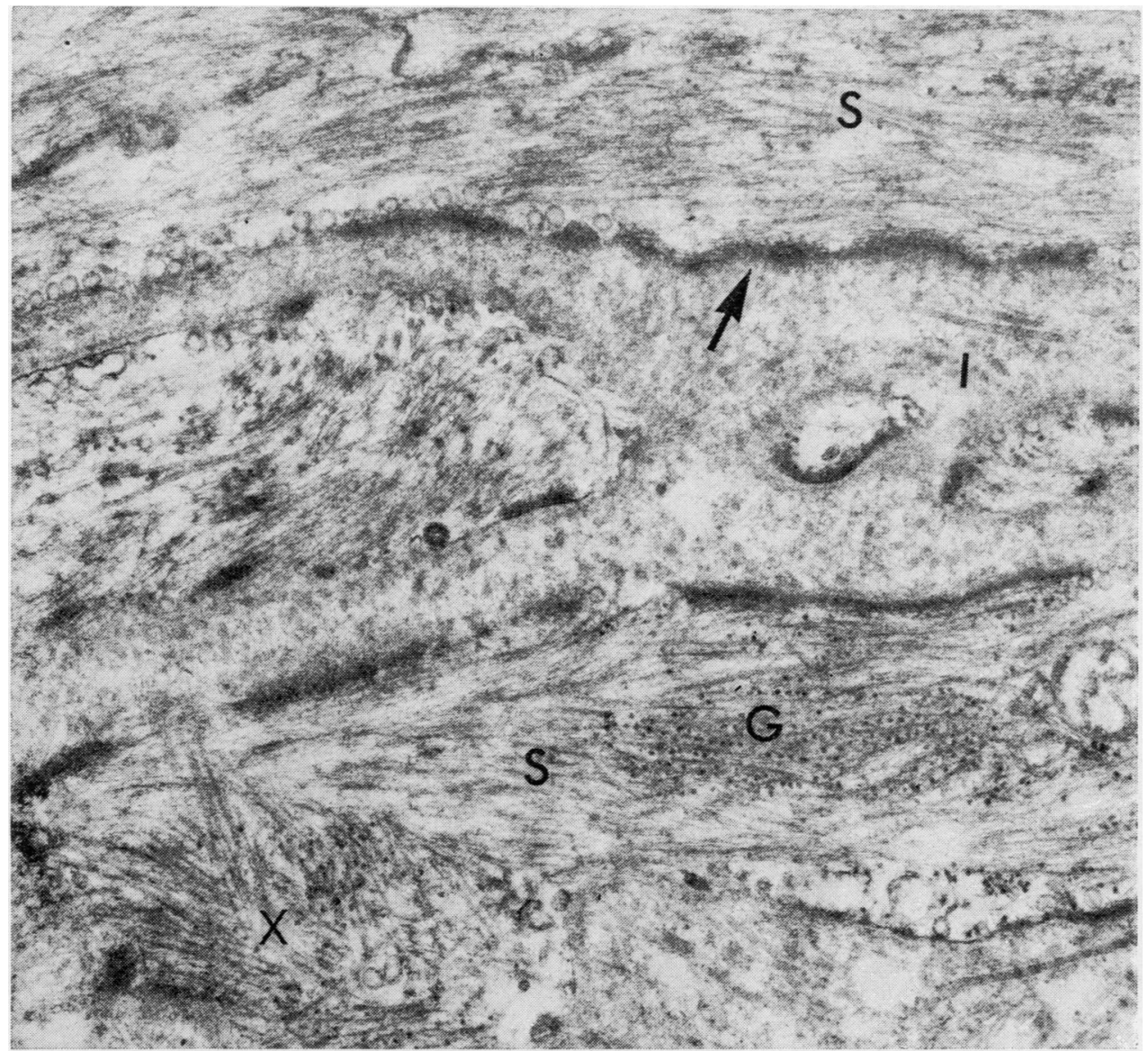

Fig. 5 Oesophagus. Longitudinally sectioned smooth muscle cells $(S)$ show disorientated thick filaments $(X)$. Cytoplasmic densities are seen along the plasma cell membrane (thick arrow). $G=g l y c o g e n$ body; $I=$ interstitial space. $\times 28500$ 
and a low-density, non-homogenous material occupied the interstitial space between the muscle cells (Fig. 5). The mitochondria were swollen, and their cristae were disrupted; this may be related to postmortem changes.

\section{Discussion}

There have been few pathological reports on cardiac involvement in myotonic dystrophy. Vacuolisation of the sarcoplasmic reticulum (Bulloch et al., 1967), an increase in interstitial tissue (Church, 1967), and fatty infiltration (Bulloch et al., 1967; Church, 1967) are some of the lesions encountered. Recently, Rausing (1972) described foci of myocarditis in a mother and her son. Such a lesion and other degenerative processes may explain the enhanced excitability of the myocardial tissue in myotonia dystrophica.

The main changes in the myocardium of our patient were extensive accumulation of mitochondria and dense intramitochondrial granules. Intramitochondrial electron-dense bodies are known to occur in experimentally induced cardiac necrosis (Kovacs et al., 1972), in ischaemic injury of the myocardium (Jennings et al., 1969), or in drug-induced injury (D'Agostino, 1963; Maruffo, 1967). Dense inclusions were also described in alcoholic cardiomyopathy (Hibbs et al., 1965) and are believed to be evidence of mitochondrial degeneration. To our knowledge, intramitochondrial granules have not been reported in the hearts of patients with myotonia dystrophica. The possibility that the mitochondrial changes are related to autolytic processes should be considered.

In this study the ultrastructural features of skeletal muscle were similar to those described by others (Aleu and Afifi, 1964; Klinkerfuss, 1967; Schotland, 1970). No important changes of the sarcotubular system were observed in the present case. The consecutive examination of the skeletal muscle enabled us to evaluate the progress of significant muscle lesions. In the 33 months' interval between the first and second examinations, severe atrophy and degenerative changes occurred in all skeletal muscles. Whereas in the first examination satellite cells were frequent, none was noticed in the second specimen, which was more severely affected. Bulloch et al. (1967), Schotland (1970), and Klinkerfuss (1967) mentioned the presence of satellite cells in early and moderately advanced cases of myotonic dystrophy. Aleu and Afifi (1964) commented on the lack of satellite cells, but they do not include a clinical report of their patients and we do not know how advanced the disease had been. The presence or absence of satellite cells in the muscles of myotonic patients may be of prognostic value in evaluating the progress of the disease.

In a survey of 16 patients with myotonic dystrophy (Harvey et al., 1965) widespread involvement of the cardiac and smooth muscles was noted. Based on manometric, radiological, and motility studies, the authors concluded that, in addition to the gastrointestinal tract, there was involvement of the smooth muscle of the gallbladder and of the urinary bladder. Pruzanski and Huvos (1967) revealed histologically a moderate decrease in the size of the intestinal smooth muscle fibres. The width of smooth muscle cells varies considerably according to their location, and in the human colon is said to be between 3 and $10 \mu \mathrm{m}$ (Matthews and Martin, 1973). It appears that, in our material, the thickness of the oesophageal and intestinal smooth muscle cells was at the lowest level for a normal smooth muscle cell. Degenerative focal lesions were clearly demonstrated in the oesophageal smooth muscle cells in areas of glycogen accumulation and of disorientated filaments.

No abnormalities of the smooth muscle cells of the aortic media were detected, except for a paucity of pinocytotic vesicles. The sheets of dense material that occupied much of the space between smooth muscle cells contained fibrin-like material and suggested alterations of the elastic material. These changes may or may not be related to the atherosclerotic lesion present in our patient.

Summarising the extent of the damage to the three kinds of muscle - skeletal, myocardial, and smoothit seems that the striated skeletal muscle was affected more than the other types of muscle. Degenerative mitochondrial changes were seen in the myocardium. Mild degenerative changes were encountered in the smooth muscle cells of the oesophagus.

Our thanks are due to Mrs Z. Weinburg and Mrs A. Ezioni for skilled technical assistance.

\section{References}

Aleu, F. P., and Afifi, A. K. (1964). Ultrastructure of muscle in myotonic dystrophy. American Journal of Pathology, 45, 221-225.

Bulloch, R. T., Davis, J. L., and Hara, M. (1967). Dystrophia myotonica with heart block: a light and electron microscopic study. Archives of Pathology, 84, $130-140$

Cannon, P. J. (1962). The heart and lungs in myotonic muscular dystrophy. American Journal of Medicine, 32, 765-775.

Church, S. C. (1967). The heart in myotonia atrophica. Archives of Internal Medicine, 119, 176-181.

D'Agostino, A. N. (1963). An electron microscopic study of skeletal and cardiac muscle of the rat poisoned by plasmocid. Laboratory Investigation, 12, 1060-1071.

Daniel, P. M., and Strich, S. J. (1964). Abnormalities in 
the muscle spindles in dystrophia myotonica. Neurology (Minneapolis), 14, 310-316.

Harvey, J. C., Sherbourne, D. H., and Siegel, C. I. (1965). Smooth muscle involvement in myotonic dystrophy. American Journal of Medicine, 39, 81-90.

Hibbs, R. G., Ferrans, V. J., Black, W. C., Weilbaecher, D. G., Walsh, J. J., and Burch, G. E. (1965). Alcoholic cardiomyopathy: an electron microscopic study. American Heart Journal, 69, 766-779.

Jennings, R. B., Sommers, H. M., Herdson, P. B., and Kaltenbach, J. P. (1969). Ischemic injury of myocardium. Annals of the New York Academy of Sciences, 156, 61-78.

Klinkerfuss, G. H. (1967). An electron microscopic study of myotonic dystrophy. Archives of Neurology, 16, 181-193.

Kovacs, K., Gardell, C., and Blascheck, J. A. (1972). Ultrastructural studies of various forms of experimentally induced cardiac necroses. Recent Advances in Studies on Cardiac Structure and Metabolism, 1, 551-562.

Ludatscher, R., Amikam, S., and Gellei, B. (1976). Ultrastructural changes in striated muscle in myotonia dystrophica. (In Hebrew.) Harefuah, 90, 527-529.

Maas, O., and Zondek, H. (1920). Untersuchungsbefund an einem Fall von Dystrophia myotonica. Zeitschrift für Neurologie und Psychiatrie, 59, 322-331.

Maruffo, C. A. (1967). Fine structural study of myocardial changes induced by isoproterenol in rhesus monkeys (Macaca mulatta). American Journal of Pathology, 50, 27-37.

Matthews, J. L., and Martin, J. H. (1973). Atlas of Human
Histology and Ultrastructure, 2nd edition, p. 112. Kimpton, London; Lea and Febiger, Philadelphia.

Maynard, J. A., Cooper, R. R., and Ionaescu, V. V. (1977). An ultrastructure investigation of intrafusal muscle fibers in myotonic dystrophy. Virchows Archiv A: Pathological Anatomy and Histology, 373, 1-13.

Mussini, I., Di Mauro, S., and Angelini, C. (1970). Early ultrastructural and biochemical changes in muscle in dystrophia myotonica. Journal of Neurological Sciences, 10, 585-604.

Pruzanski, W., and Huvos, A. G. (1967). Smooth muscle involvement in primary muscle disease I. Myotonic dystrophy. Archives of Pathology, 83, 229-233.

Rausing, A. (1972). Focal myocarditis in familial dystrophia myotonica. British Heart Journal, 34, 12921294.

Schotland, D. L. (1970). An electron microscopic investigation of myotonic dystrophy. Journal of Neuropathology and Experimental Neurology, 29, 241-253.

Swash, M., and Fox, K. P. (1975). Abnormal intrafusal muscle fibers in myotonic dystrophy: a study using serial sections. Journal of Neurology, Neurosurgery and Psychiatry, 38, 91-99.

Uemura, N., Tanaka, H., Niimura, T., Hashiguchi, N., Yoshimura, M., Terashi, S., and Kanehisa, T. (1973). Electrophysiological and histological abnormalities of the heart in myotonic dystrophy. American Heart Journal, 86, 616-624.

Requests for reprints to: Dr Ruth Ludatscher, Departe ment of Pathology, The Technion School of Medicine 12 Haaliya Street, Bat-Galim, POB 9649, Haifa, Israel. 